\title{
Design of hybrid controller for synchronization control of Chen chaotic system
}

\author{
Xiaoyu Zhang ${ }^{\mathrm{a}}$, Xiaodi Lij ${ }^{\mathrm{a}, \mathrm{b}, *}$, Xiuping Han ${ }^{\mathrm{a}}$ \\ a School of Mathematics and Statistics, Shandong Normal University, Ji'nan, 250014, P. R. China. \\ ${ }^{b}$ Institute of Data Science and Technology, Shandong Normal University, Ji'nan, 250014, P. R. China. \\ Communicated by R. Saadati
}

\begin{abstract}
This paper deals with the synchronization control of Chen chaotic system using a hybrid control which includes continuous state feedback control, the impulsive control and the nonlinear feedback law. To this end, a hybrid controller based on linear matrix inequality (LMI) and average dwell time (ADT) is derived by employing impulsive control theory. The main advantage of the result lies in that, for one thing, they are complementary to each other, that is, when the impulse inputs occur in terms of disturbances which do harm to the synchronization, the continuous state feedback control will cover the weakness and stabilize the error system, and conversely, when the continuous state feedback control is given in terms of external disturbances which do harm to the synchronization, the impulsive control input will stabilize the error system; for another, the developed result is based on ADT condition and dropped the restriction on the upper and lower bounds of the impulsive intervals. Finally, numerical simulations are presented to show the effectiveness of the proposed chaos synchronization scheme. (C) 2017 All rights reserved.
\end{abstract}

Keywords: Chen system, impulsive control, synchronization, average dwell-time (ADT), hybrid controller. 2010 MSC: 34K35, 34D06, 34K34.

\section{Introduction}

Chaos is an interesting phenomenon in nonlinear dynamical systems. Since Edward Lorenz found the first chaotic attractor in a three-dimensional system [26], the chaos theory has been intensively researched because of its wide applications in various areas such as secure communication [33], neural networks [16], laser physics [30] and chemical reactor[9], finance [1], economy [13], etc.. Chaotic systems are nonlinear aperiodic systems with high sensitivity to initial conditions which leads to unpredictability of its long term dynamic behavior. Chen system, was found by Chen and Ueta in 1999 [2], which is similar but topological nonequivalent to Lorenz-like systems, such as Chua's circuit [3], Lü system [22], and the Lorenz system family [20].

Chaos synchronization has been widely studied over decades since it was first proposed by Pecora and Carrol in 1990 [24]. Generally, chaos synchronization is to design a system to imitate the dynamical

\footnotetext{
*Corresponding author

Email address: lxd@sdnu.edu.cn (Xiuping Han)
}

doi:10.22436/jnsa.010.06.41 
behavior of another chaotic system, that is, the dynamic behavior of the designed system must converge to the chaotic behavior. The system with coupled inputs is called the slave (response) system, which is designed to synchronize the chaotic behavior of a system called the master (drive) system. Lots of methods have been proposed to achieve the synchronization of chaotic systems, such as adaptive control [21, 27], fuzzy control [34], backstepping control [11, 25, 31], sliding mode control [8], digital redesign control [4], and impulsive control [15, 17].

As we know, impulsive control as a class of discontinuous control methods has been widely used to synchronize and stabilize various systems [6, 10, 14, 18, 19, 23, 35]. For example, Lu et al. [23] studied the globally exponential synchronization of impulsive dynamical networks in which the synchronizing impulses and desynchronizing impulses were considered; Hespanha et al. [6] considered the input-to-state stability of impulsive systems based on ADT method and $[19,35]$ handled with input-to-state stability of systems with delayed impulses; Li et al. [14] dealt with the complete synchronization of delayed chaotic neural networks by intermittent control with two switches in a control period. The main thought of impulsive control is changing the states of continuous dynamic systems by discontinuous control input at certain time instants. Impulsive control is effective and robust, from the control point of view, since only small control gains are needed [12]. Moreover, impulsive control has great advantages over general continuous control schemes due to reduced control cost and simple structure [29]. Furthermore, impulsive control can greatly reduce the information redundancy of the transmitted signal and increase robustness of the system against the disturbances [5, 28, 32]. Sometimes when the continuous control input does not work well, even for the case that there exists external disturbances, we can use the impulsive control to cover the weakness and achieve the desired performance; conversely, if the impulse inputs occur in terms of disturbances instead of the control effect, we can use the continuous control to balance it. Hence, the hybrid control including continuous control and impulsive control is of great significance.

This paper investigates the synchronization control of Chen chaotic system via a hybrid controller. The controller proposed consists of continuous state feedback controller, impulsive controller and the nonlinear feedback law. Especially, the continuous state feedback controller and the impulsive controller are complementary to each other, when one of them does not work well, the other one will balance it. In addition, we develop the ADT [7, 19, 35] method to the design of hybrid controller and drop the restriction on the lower and upper bounds of the impulsive intervals. The rest of the paper is organized as follows. Section 2 briefly introduces some notations and preliminary works. Section 3 presents the control laws to ensure the synchronization of Chen system via the proposed hybrid controller. Section 4 presents numerical examples of the proposed control laws. Concluding remarks are given in Section 5.

\section{Preliminaries}

Notations. Let $\mathbb{R}$ denote the set of real numbers, $\mathbb{R}^{n}$ and $\mathbb{R}^{n \times m}$ the $n$-dimensional and $n \times m$-dimensional real spaces equipped with the Euclidean norm $|\cdot|$, respectively, $\mathbb{Z}_{+}$the set of positive integer numbers, and $A>0$ or $A<0$ denotes that the matrix $A$ is a symmetric and positive or negative definite matrix. The impulse time sequence $\left\{t_{n}\right\} \in \digamma$ if it satisfies $0 \leqslant t_{0}<t_{1}<\cdots<t_{k} \rightarrow+\infty$ as $k \rightarrow+\infty$. Let $N(t, s)$ denote the number of the impulsive time instances in the semi-open time interval $[s, t)$, and I denotes the identity matrix with appropriate dimension. Moreover, the notation $\star$ denotes the symmetric block in one symmetric matrix.

Chen system which was created by Chen and Ueta in 1999 [2], can be described by the following set of ordinary differential equations

$$
\left\{\begin{array}{l}
\dot{x_{1}}=a\left(x_{2}-x_{1}\right) \\
\dot{x_{2}}=(c-a) x_{1}-x_{1} x_{3}+c x_{2} \\
\dot{x_{3}}=x_{1} x_{2}-b x_{3}
\end{array}\right.
$$

where $x_{1}, x_{2}, x_{3}$ are state variables and $a, b, c$ are system parameters. When $a=35, b=3$, and $c=28$, the system (2.1) exhibits chaotic behavior [2]. 
To investigate the synchronization control of the Chen chaotic systems, we choose (2.1) as the drive system and the corresponding response system is given by

$$
\left\{\begin{array}{l}
\dot{y}_{1}=a\left(y_{2}-y_{1}\right)+u_{1}, t \neq t_{n} \\
\dot{y} 2=(c-a) y_{1}-y_{1} y_{3}+c y_{2}+u_{2}+\alpha(t), t \neq t_{n} \\
\dot{y} \dot{y}_{3}=y_{1} y_{2}-b y_{3}+u_{3}+\beta(t), t \neq t_{n} \\
y(t)=u_{0}\left(t^{-}\right)+x\left(t^{-}\right), t=t_{n}
\end{array}\right.
$$

where $u=\left(u_{1}, u_{2}, u_{3}\right)^{T}$ is the state feedback control input or external disturbance when $t \neq t_{n} ; u_{0}$ is the impulsive control input or impulsive perturbation when $t=t_{n} ; \alpha(t), \beta(t)$ are the secondary control input to deal with nonlinearity in the system.

Define the error variable as $e(t)=\left(e_{1}, e_{2}, e_{3}\right)^{T}, e_{i}=y_{i}-x_{i}, i=1,2,3$. We consider $u(t)=K e(t)$, $K=\left(k_{i j}\right)_{3 \times 3}$, i.e., $u_{i}(t)=k_{i 1} e_{1}+k_{i 2} e_{2}+k_{i 3} e_{3}, i=1,2,3$ and $u_{0}=k_{0} e\left(t_{k}^{-}\right), k_{0} \in \mathbb{R}^{3 \times 3}$. Then the error system can be rewritten as

$$
\left\{\begin{array}{l}
\dot{e}(t)=A e(t)+f(t, e)+B u(t)+G(t), t \neq t_{n} \\
e(t)=K_{0} e\left(t^{-}\right), t=t_{n}
\end{array}\right.
$$

where

$$
\begin{aligned}
A & =\left[\begin{array}{ccc}
-a & a & 0 \\
c-a & c & 0 \\
0 & 0 & -b
\end{array}\right], B=\left[\begin{array}{lll}
1 & 0 & 0 \\
0 & 1 & 0 \\
0 & 0 & 1
\end{array}\right], u(t)=\left[\begin{array}{c}
u_{1} \\
u_{2} \\
u_{3}
\end{array}\right], G(t)=\left[\begin{array}{c}
0 \\
\alpha(t) \\
\beta(t)
\end{array}\right], \\
f(t, e) & =\left[\begin{array}{c}
0 \\
-y_{1} y_{3} \\
y_{1} y_{2}
\end{array}\right]-\left[\begin{array}{c}
0 \\
-x_{1} x_{3} \\
x_{1} x_{2}
\end{array}\right]=\left[\begin{array}{c}
0 \\
-y_{1} e_{3}-x_{3} e_{1} \\
y_{1} e_{2}+x_{2} e_{1}
\end{array}\right] .
\end{aligned}
$$

Definition 2.1. Systems (2.1) and (2.2) are said to be exponentially synchronized if there exist constants $\lambda>0$ and $M \geqslant 1$ such that $|e(t)| \leqslant M\left|x_{0}-y_{0}\right| e^{-\lambda t}$ for all $t>0$, where $x_{0}$ and $y_{0}$ are the initial values of the state variables $x$ and $y$, respectively. Here, $\lambda$ is called the convergence rate (or degree) of exponential synchronization.

\section{Main results}

The purpose of this section is to construct a hybrid controller for the error system (2.3) such that it is globally exponential stable. The controller consists of three parts. One part is the continuous state feedback controller $u$, the secondary is the nonlinear feedback law $G$ for handling the nonlinear term, and the third one is the impulsive controller $\mathfrak{u}_{0}$. First, we design the secondary control input $G$ as

$$
G(t)=\left[\begin{array}{c}
0 \\
\alpha(t) \\
\beta(t)
\end{array}\right]=\left[\begin{array}{c}
0 \\
y_{1} e_{3}+x_{3} e_{1} \\
-y_{1} e_{2}-x_{2} e_{1}
\end{array}\right]
$$

Then to find out the state feedback control gain $\mathrm{K}$ and the impulsive control gain $\mathrm{K}_{0}$, we present the following theorem.

Theorem 3.1. Assume that there exist matrices $P>0, W, W_{0} \in \mathbb{R}^{3 \times 3}$, constants $\rho \in \mathbb{R}, d \in \mathbb{R}$ such that

(i) $A^{T} P+P A+W+W^{T}+\rho P \leqslant 0 ;$ 
(ii) $\left[\begin{array}{cc}-e^{-\mathrm{d}} \mathrm{P} & \mathrm{W}_{0}^{\mathrm{T}} \\ \star & -\mathrm{P}\end{array}\right] \leqslant 0$;

(iii) for any constants $\mathrm{c}>0, \lambda>0$, let $\digamma[\mathrm{c}, \lambda]$ denote the class of impulse time sequence $\left\{\mathrm{t}_{\mathrm{n}}\right\}$ satisfying

$$
-\mathrm{dN}(\mathrm{t}, \mathrm{s})-(\rho-\lambda)(\mathrm{t}-\mathrm{s}) \leqslant \mathrm{c}, \quad \forall \mathrm{t} \geqslant s \geqslant \mathrm{t}_{0} .
$$

Then the error system (2.3) is globally exponentially stable over class $F[c, \lambda]$, that is, the drive system (2.1) can be exponentially synchronized by the response system (2.2), where control gains are designed by

$$
\mathrm{K}=\mathrm{P}^{-1} \mathrm{~W}, \quad \mathrm{~K}_{0}=\mathrm{P}^{-1} \mathrm{~W}_{0} .
$$

Proof. Choose the candidate Lyapunov function be in the form of

$$
V(t)=e^{T}(t) \operatorname{Pe}(t) .
$$

The derivative of $V(t)$ along the solutions of the error system is given by

$$
\begin{aligned}
\dot{V}(t) & =\dot{e}^{T}(t) P e(t)+e^{T}(t) P \dot{e}(t) \\
& =e^{T}(t)(A+K)^{T} P e(t)+e^{T}(t) P(A+K) e(t) \\
& =e^{T}(t)\left(P A+A^{T} P+P K+K^{T} P\right) e(t) \\
& \leqslant-\rho V(t), t \neq t_{n}, \\
V\left(t_{n}\right) & =e^{T}\left(t_{n}\right) P e\left(t_{n}\right) \\
& =e^{T}\left(t_{n}^{-}\right) \bar{K}^{T} P \bar{K} e\left(t_{n}^{-}\right) \\
& \leqslant e^{-d} e^{T}\left(t_{n}^{-}\right) \operatorname{Pe}\left(t_{n}^{-}\right) \leqslant e^{-d} V\left(t_{n}^{-}\right) .
\end{aligned}
$$

It then follows from (i) and (ii) that

$$
\begin{cases}\dot{V}(t) \leqslant-\rho V(t), & t \neq t_{n} \\ V(t) \leqslant e^{-d} V\left(t^{-}\right), & t=t_{n}\end{cases}
$$

When $t \in\left[t_{0}, t_{1}\right)$, it follows that

$$
\mathrm{V}(\mathrm{t}) \leqslant \mathrm{e}^{-\rho\left(\mathrm{t}-\mathrm{t}_{0}\right)} \mathrm{V}\left(\mathrm{t}_{0}\right), \quad \mathrm{V}\left(\mathrm{t}_{1}^{-}\right) \leqslant \mathrm{e}^{-\rho\left(\mathrm{t}_{1}-\mathrm{t}_{0}\right)} \mathrm{V}\left(\mathrm{t}_{0}\right) .
$$

When $t \in\left[t_{1}, t_{2}\right)$, we have

$$
\begin{aligned}
V\left(t_{1}\right) & \leqslant e^{-d} e^{-\rho\left(t_{1}-t_{0}\right)} V\left(t_{0}\right), \\
V(t) & \leqslant e^{-\rho\left(t-t_{1}\right)} V\left(t_{1}\right) \leqslant e^{-d} e^{-\rho\left(t_{1}-t_{0}\right)} V\left(t_{0}\right) e^{-\rho\left(t_{1}-t_{0}\right)} V\left(t_{0}\right), \\
V\left(t_{2}^{-}\right) & \leqslant e^{-d} e^{-\rho\left(t_{2}-t_{0}\right)} V\left(t_{0}\right) .
\end{aligned}
$$

By simple induction, it can be deduced that

$$
V(t) \leqslant e^{-n d} e^{-\rho\left(t-t_{0}\right)} V\left(t_{0}\right), t \in\left[t_{n}, t_{n+1}\right),
$$

which together with (3.2) yields that

$$
V(t) \leqslant e^{c-\lambda\left(t-t_{0}\right)} V\left(t_{0}\right), t \geqslant t_{0} .
$$

It implies that the error system (2.3) is globally exponentially stable at the origin, that is, the drive system (2.1) is synchronized by the response system (2.2). 
Remark 3.2. One may observe from Theorem 3.1 that we do not impose any positive or negative restriction on constants $\rho$ and $d$, which implies that it admits the existence of not only the synchronizing impulses and desynchronizing impulses, but also the synchronizing continuous state feedback and desynchronizing continuous state feedback. Exactly speaking, when $\mathrm{d}<0$, the impulses can potentially destroy the stability of the error system, and usually we require that they do not happen too frequently. Note that condition (3.2) enforces an upper bound on the number of impulses times, but it may be large enough since that it is $\rho$-dependent. Because the continuous state feedback is imposed to achieve the stabilization of error system through condition (i), which will be helpful to the choice of constant $\rho$. Conversely, when $d>0$, the impulses can potentially benefit the stability of the error system. In this case, when there is no continuous state feedback or the external input is a kind of disturbances, the impulsive control can be used to stabilize the error system and achieve the synchronization. By the above discussions, it can be analytically concluded that our synchronization criterion is less conservative than the results in $[5,6,12,14,15,23,29,32]$.

Assume that $u(t)$ is not the control input but the bounded external disturbance which maybe destroy the synchronization, that is, assume that $u(t)=K e(t), K$ is pre-given. Based on Theorem 3.1 and the average dwell-time (ADT) analysis method for switched systems proposed by Hespanha and Morse [7], one may derive the following ADT-like conditions.

Corollary 3.3. If there exist matrices $\mathrm{P}>0, \mathrm{~W}_{0} \in \mathbb{R}^{3 \times 3}$, constants $\rho \in \mathbb{R}_{-}$and $\mathrm{d} \in \mathbb{R}_{+}$such that

(i) $A^{T} P+K^{T} P+P A+P K+\rho P \leqslant 0 ;$

(ii) $\left[\begin{array}{cc}-e^{-\mathrm{d} P} & \mathrm{~W}_{0}^{\mathrm{T}} \\ \star & -\mathrm{P}\end{array}\right] \leqslant 0$,

and let $\digamma_{\mathrm{r}-\mathrm{avg}}\left[\mathrm{N}_{0}, \tau^{\star}\right] \subseteq \digamma$ denote the class of reverse average dwell-time impulse time sequence satisfying

$$
\mathrm{N}(\mathrm{t}, \mathrm{s}) \geqslant \mathrm{N}_{0}+\frac{\mathrm{t}-\mathrm{s}}{\tau^{\star}}, \quad \forall \mathrm{t} \geqslant \mathrm{s} \geqslant \mathrm{t}_{0},
$$

where $\mathrm{N}_{0}>0, \tau^{\star}<\frac{\mathrm{d}}{|\rho|}$, then the drive system (2.1) can be synchronized by the response system (2.2) over the class $\digamma_{\mathrm{r}-\mathrm{avg}}\left[\mathrm{N}_{0}, \tau^{\star}\right]$. The impulsive control gain is designed by

$$
\mathrm{K}_{0}=\mathrm{P}^{-1} \mathrm{~W}_{0} .
$$

On the other hand, we consider the case that $u_{0}(t)$ is not the impulsive control input but the bounded impulsive perturbations, assume that $\mathrm{u}_{0}(t)=\mathrm{K}_{0} e, \mathrm{~K}_{0}$ is pre-given. Then based on Theorem 3.1, we can design the continuous state feedback controller $u$ as follows.

Corollary 3.4. If there exist matrices $\mathrm{P}>0, \mathrm{~W} \in \mathbb{R}^{3 \times 3}$, constants $\rho \in \mathbb{R}_{+}$and $\mathrm{d} \in \mathbb{R}_{\text {- such that }}$

(i) $A^{T} P+P A+W+W^{T}+\rho P \leqslant 0 ;$

(ii) $\left[\begin{array}{cc}-e^{-\mathrm{d} P} & \mathrm{~K}_{0}^{\mathrm{T}} \mathrm{P} \\ \star & -\mathrm{P}\end{array}\right] \leqslant 0$,

and let $\digamma_{\text {avg }}\left[\mathrm{N}_{0}, \tau^{\star}\right] \subseteq \digamma$ denote the class of average dwell-time (ADT) impulse time sequence satisfying

$$
\mathrm{N}(\mathrm{t}, \mathrm{s}) \leqslant \mathrm{N}_{0}+\frac{\mathrm{t}-\mathrm{s}}{\tau^{\star}}, \quad \forall \mathrm{t} \geqslant \mathrm{s} \geqslant \mathrm{t}_{0}
$$

where $\mathrm{N}_{0}>0, \tau^{\star}>\frac{|\mathrm{d}|}{\rho}$, then the drive system (2.1) can be synchronized by the response system (2.2) over the class $\digamma_{\text {avg }}\left[\mathrm{N}_{0}, \tau^{\star}\right]$. The continuous state feedback control gain is designed by

$$
\mathrm{K}=\mathrm{P}^{-1} \mathrm{~W} \text {. }
$$

Corollary 3.5. Assume that there exist matrices $P>0, W, W_{0} \in \mathbb{R}^{3 \times 3}$, and constants $\rho \in \mathbb{R}_{+}$and $\mathrm{d} \in \mathbb{R}_{+}$such that (i) and (ii) hold. Then the drive system (2.1) can be synchronized by the response system (2.2) for any impulsive sequence. 


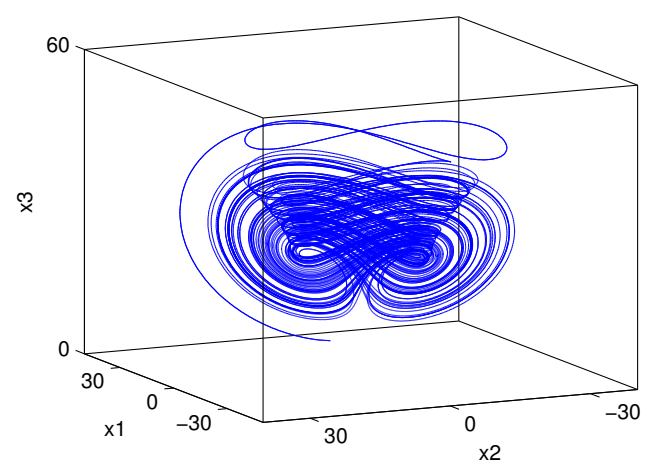

Figure 1: The chaotic behavior of the Chen system (2.1) with $a=35, b=3$, and $c=28$.

\section{Numerical simulations}

We consider the Chen system (2.1) with $a=35, b=3$, and $c=28$. Fig. 1 shows the chaotic behavior. Assume that the nonlinear feedback law G is given in (3.1). Now we consider the synchronization control of systems (2.1) and (2.2) with three cases.

Case I. Hybrid control. We will design the continuous state feedback controller $u$ and the impulsive controller $u_{0}$. In this case, we choose $\rho=1$ and $d=0.48$. Then the LMIs in Theorem 3.1 have feasible solutions and the control gains $\mathrm{K}$ and $\mathrm{K}_{0}$ can be derived such as

$$
\mathrm{K}=\left[\begin{array}{ccc}
34.0458 & -18.3778 & 0 \\
-9.6222 & -28.9542 & 0 \\
0 & 0 & 2.0458
\end{array}\right], \mathrm{K}_{0}=0.3028 \mathrm{I}
$$

Then based on the hybrid control, the error system (2.3) is globally exponentially stable, see Fig. 2 (a).
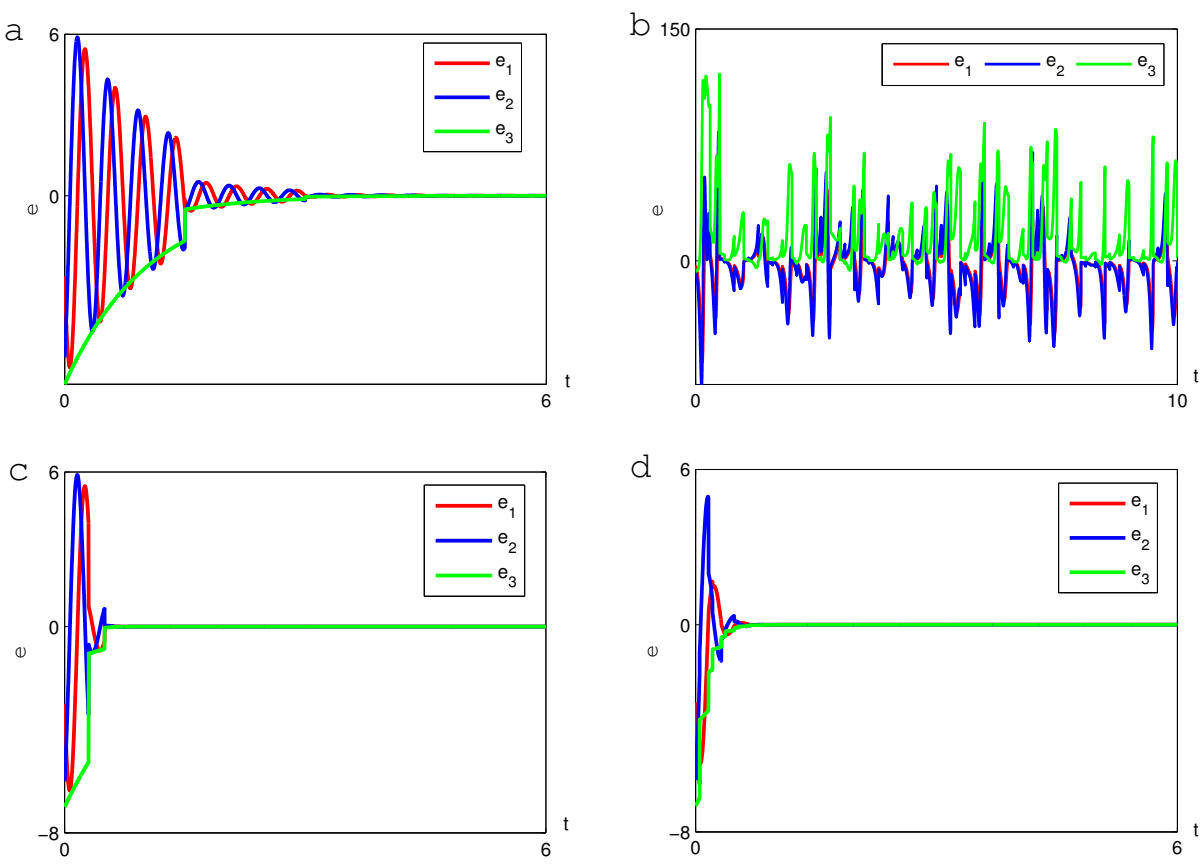

Figure 2: (a). Trajectories of error variable $e_{i}$ based on the hybrid control; (b). Trajectories of error variable $e_{i}$ when there is no control input (i.e., $u=0$ ) but with the impulsive perturbation $u_{0}$; (c). Trajectories of error variable $e_{i}$ when there is continuous state feedback control $u ;(d)$. Trajectories of error variable $e_{i}$ with impulsive control input $u_{0}$. 
Case II. Continuous feedback control. $u$ is the control input which will be designed and $u_{0}$ is the impulsive perturbation. Assume that $\mathrm{u}_{0}=\mathrm{K}_{0} e, \mathrm{~K}_{0}=0.2 \mathrm{I}$, is the impulsive perturbation. The impulsive sequence is considered by

$$
\mathrm{t}_{4 \mathrm{n}-3}=2 \mathrm{n}-1.9, \mathrm{t}_{4 \mathrm{n}-2}=2 \mathrm{n}-1.7, \mathrm{t}_{4 \mathrm{n}-1}=2 \mathrm{n}-1, \mathrm{t}_{4 \mathrm{n}}=2 \mathrm{n},
$$

$n \in \mathbb{Z}_{+}$, which satisfies $\tau^{\star}=0.5>0.48=|d| / \rho$. In this case, when there is no control input (i.e., $u=0$ ), the error system (2.3) with impulsive perturbation $\mathfrak{u}_{0}$ is not stable, see Fig. $2(b)$. We consider $\rho=1$, $\mathrm{d}=-0.5$, then it is easy to derive that

$$
K=\left[\begin{array}{ccc}
33.3906 & -8.3349 & 0 \\
-19.6651 & -29.6094 & 0 \\
0 & 0 & 1.3906
\end{array}\right]
$$

Then the error system with control gain $\mathrm{K}$ is globally exponentially stable, see Fig. 2 (c).

Case III. Impulsive control input. When there is no continuous control input, i.e., $\mathbf{u}=0$, we can design the impulsive controller. Consider the impulsive sequence

$$
\mathrm{t}_{2 \mathrm{n}-1}=0.16 \mathrm{n}-0.11, \mathrm{t}_{2 \mathrm{n}}=0.16 \mathrm{n}, \mathrm{n} \in \mathbb{Z}_{+},
$$

which satisfies $\tau^{\star}=0.08<0.0833=\mathrm{d} /|\rho|$. Choose $\rho=-48$ and $\mathrm{d}=4$, then the control gain can be derived by

$$
\mathrm{K}_{0}=\left[\begin{array}{ccc}
0.8440 & 0.0422 & 0 \\
0.0549 & 0.3937 & 0 \\
0 & 0 & 0.5506
\end{array}\right]
$$

Then the error system is globally exponentially stable, see Fig. 2 (d).

\section{Conclusion}

The synchronization problem of Chen chaotic systems via a hybrid controller was investigated in this paper. The proposed hybrid controller which combined the continuous state feedback controller, the impulsive controller, and the nonlinear feedback law was derived, which was presented in terms of LMI and ADT conditions. Finally, numerical simulations were provided to show the effectiveness of the proposed method. In our study, we note that the ADT like condition is based on the fact that the nonlinear feedback law is pre-given, but it is unclear whether the conditions derived can be developed to the cases that there is no nonlinear feedback. Investigating this issue is a topic for future research. Another topic is to develop the hybrid controller, especially ADT like method, in this paper, to delayed chaotic systems.

\section{Acknowledgment}

This work was supported by National Natural Science Foundation of China $(61673247,11301308)$, Natural Science Foundation of Shandong Province (Grant No. ZR2013AQ008) and the outstanding Youth Foundation of Shandong Province (ZR2016JL024).

\section{References}

[1] R. Carrasco, M. Vargas, M. Alfaro, I. Soto, G. Fuertes, Copper metal price Using chaotic time series forecating, IEEE Lat. Am. Trans., 13 (2015), 1961-1965. 1

[2] G.-R. Chen, T. Ueta, Yet another chaotic attractor, Internat. J. Bifur. Chaos Appl. Sci. Engrg., 9 (1999), 1465-1466. 1, 2,2

[3] L. O. Chua, M. Komuro, T. Matsumoto, The double scroll family, I, Rigorous proof of chaos, IEEE Trans. Circuits and Systems, 33 (1986), 1072-1097.1 
[4] S.-M. Guo, L. S. Shieh, G.-R. Chen, C.-F. Lin, Effective chaotic orbit tracker: a prediction-based digital redesign approach, IEEE Trans. Circuits Systems I Fund. Theory Appl., 47 (2000), 1557-1560. 1

[5] W. M. Haddad, V. Chellaboina, S. G. Nersesov, Impulsive and hybrid dynamical systems. Stability, dissipativity, and control, Princeton Series in Applied Mathematics, Princeton University Press, Princeton, NJ, (2006). 1, 3.2

[6] J. P. Hespanha, D. Liberzon, A. R. Teel, Lyapunov conditions for input-to-state stability of impulsive systems, Automatica J. IFAC, 44 (2008), 2735-2744. 1, 3.2

[7] J. P. Hespanha, A. S. Morse, Stability of switched systems with average dwell-time, Proceedings of the 38th IEEE Conference on Decision and Control, Phoenix, AZ, USA, 3 (1999), 2655-2660. 1, 3

[8] T.-Y. Jing, F.-Q. Chen, X.-H. Zhang, Finite-time lag synchronization of time-varying delayed complex networks via periodically intermittent control and sliding mode control, Neurocomputing, 199 (2016), 178-184. 1

[9] H. Kheiri, B. Naderi, Dynamical behavior and synchronization of chaotic chemical reactors model, Iran. J. Math. Chem., 6 (2015), 81-92. 1

[10] X.-D. Li, M. Bohner, C.-K. Wang, Impulsive differential equations: periodic solutions and applications, Automatica J. IFAC, 52 (2015), 173-178. 1

[11] D. Li, J.-D. Cao, Finite-time synchronization of coupled networks with one single time-varying delay coupling, Neurocomputing, 166 (2015), 265-270. 1

[12] X.-D. Li, J.-D. Cao, An impulsive delay inequality involving unbounded time-varying delay and applications, IEEE Trans. Automat. Control, 62 (2017), 3618-3625. 1, 3.2

[13] X.-D. Li, X.-L. Fu, Lag synchronization of chaotic delayed neural networks via impulsive control, IMA J. Math. Control Inform., 29 (2012), 133-145. 1

[14] Y. Li, C.-D. Li, Complete synchronization of delayed chaotic neural networks by intermittent control with two switches in a control period, Neurocomputing, 173 (2016), 1341-1347. 1, 3.2

[15] X.-D. Li, R. Rakkiyappan, Impulsive controller design for exponential synchronization of chaotic neural networks with mixed delays, Commun. Nonlinear Sci. Numer. Simul., 18 (2013), 1515-1523. 1, 3.2

[16] X.-D. Li, R. Rakkiyappan, N. Sakthivel, Non-fragile synchronization control for Markovian jumping complex dynamical networks with probabilistic time-varying coupling delays, Asian J. Control, 17 (2015), 1678-1695. 1

[17] X.-D. Li, S.-J. Song, Stabilization of delay systems: delay-dependent impulsive control, IEEE Trans. Automat. Control, 62 (2017), 406-411. 1

[18] X.-D. Li, J.-H. Wu, Stability of nonlinear differential systems with state-dependent delayed impulses, Automatica J. IFAC, 64 (2016) 63-69. 1

[19] X.-D. Li, X.-L. Zhang, S.-J. Song, Effect of delayed impulses on input-to-state stability of nonlinear systems, Automatica J. IFAC, 76 (2017), 378-382. 1

[20] X.-Y. Liang, J.-F. Zhang, X.-H. Xia, Correction to: "Adaptive synchronization for generalized Lorenz systems" [IEEE Trans. Automat. Control, 53 (2008), 1740-1746], IEEE Trans. Automat. Control, 53 (2008), 1740-1746. 1

[21] Y.-C. Liu, C.-D. Li, T.-W. Huang, X. Wang, Robust adaptive lag synchronization of uncertain fuzzy memristive neural networks with time-varying delays, Neurocomputing, 190 (2016), 188-196. 1

[22] J.-H. Lü, G.-R. Chen, A new chaotic attractor coined, Internat. J. Bifur. Chaos Appl. Sci. Engrg., 12 (2002), 659-661. 1

[23] J.-Q. Lu, D. W. C. Ho, J.-D. Cao, A unified synchronization criterion for impulsive dynamical networks, Automatica J. IFAC, 46 (2010), 1215-1221. 1, 3.2

[24] L. M. Pecora, T. L. Carroll, Synchronization in chaotic systems, Phys. Rev. Lett., 64 (1990), 821-824. 1

[25] R. Rakkiyappan, R. Sivasamy, X.-D. Li, Synchronization of identical and nonidentical memristor-based chaotic systems via active backstepping control technique, Circuits Systems Signal Process., 34 (2015) 763-778. 1

[26] C. Sparrow, The Lorenz equations: bifurcations, chaos, and strange attractors, Applied Mathematical Sciences, SpringerVerlag, New York-Berlin, (1982). 1

[27] Q.-K. Song, J.-D. Cao, Synchronization and anti-synchronization for chaotic systems, Chaos Solitons Fractals, 33 (2007), 929-939. 1

[28] I. Stamova, T. Stamov, X.-D. Li, Global exponential stability of a class of impulsive cellular neural networks with supremums, Internat. J. Adapt. Control Signal Process., 28 (2014), 1227-1239. 1

[29] J.-T. Sun, Q.-L. Han, X.-F. Jiang, Impulsive control of time-delay systems using delayed impulse and its application to impulsive master-slave synchronization, Phys. Lett. A, 372 (2008), 6375-6380. 1, 3.2

[30] R. Vicente, J. Daudén, P. Colet, R. Toral, Analysis and characterization of the hyperchaos generated by a semiconductor laser subject to a delayed feedback loop, IEEE J. Quant. Electron., 41 (2005), 541-548. 1

[31] T. Wu, M.-S. Chen, Chaos control of the modified Chua's circuit system, Phys. D, 164 (2002), 53-58. 1

[32] X.-S. Yang, J.-D. Cao, J.-L. Qiu, pth moment exponential stochastic synchronization of coupled memristor-based neural networks with mixed delays via delayed impulsive control, Neural Netw., 65 (2015), 80-91. 1, 3.2

[33] T. Yang, L. O. Chua, Impulsive stabilization for control and synchronization of chaotic systems: theory and application to secure communication, Special issue on chaos synchronization, control, and applications, IEEE Trans. Circuits Systems I Fund. Theory Appl., 44 (1997), 976-988. 1

[34] H.-T. Yau, C.-S. Shieh, Chaos synchronization using fuzzy logic controller, Nonlinear Anal. Real World Appl., 94 (2008), 1800-1810. 1

[35] X.-L. Zhang, X.-D. Li, Input-to-state stability of non-linear systems with distributed-delayed impulses, IET Control Theory Appl., 11 (2017), 81-89. 1 